\title{
The search for the host galaxy of the gamma-ray burst GRB 000214^
}

\author{
S. Guziy ${ }^{1,2}$, J. Gorosabel $^{1}$, A. J. Castro-Tirado ${ }^{1}$, A. de Ugarte Postigo ${ }^{1}$, M. Jelínek ${ }^{1}$, M. D. Pérez Ramírez ${ }^{3}$, \\ J. M. Castro Cerón ${ }^{4}$, S. Klose ${ }^{5}$, E. Palazzi ${ }^{6}$, and K. Wiersema ${ }^{7}$ \\ 1 Instituto de Astrofísica de Andalucía (IAA-CSIC), Apartado de Correos, 3004, 18080 Granada, Spain \\ e-mail: gss@iaa.es \\ 2 Nikolaev State University, Nikolska 24, 54030 Nikolaev, Ukraine \\ 3 Universidad de Jaén, Departamento de Física (EPS), Virgen de la Cabeza, 2, 23071 Jaén, Spain \\ 4 Astronomical Observatory, University of Copenhagen, Juliane Maries Vej 30, 2100 København Ø, Denmark \\ 5 Thüringer Landessternwarte Tautenburg, Sternwarte 5, 07778 Tautenburg, Germany \\ ${ }^{6}$ Istituto di Astrofisica Spaziale e Fisica Cosmica, CNR, Sezione di Bologna, via Gobetti 101, 40129 Bologna, Italy \\ 7 University of Amsterdam, Kruislaan 403, 1098 SJ Amsterdam, The Netherlands
}

Received 23 November 2004 / Accepted 3 June 2005

\section{ABSTRACT}

We present $U B V R I$-band observations taken $\sim 300$ days after the BeppoSAX $\gamma$-ray burst GRB 000214. This GRB did not show a detectable optical afterglow; however, due to the localization of a previously unknown, fading X-ray source at a tentative redshift in the range 0.37-0.47, we searched with the ESO $3.6 \mathrm{~m}$ telescope for objects with photometric redshifts consistent with the mentioned X-ray redshift range. We report four host galaxy candidates, which might be the subject of future spectroscopic observations, in order to confirm their redshifts.

Key words. gamma rays: bursts - techniques: photometric - cosmology: observations

\section{Introduction}

GRB 000214 was detected by both the GRB monitor (GRBM) and the Wide Field Cameras (WFC) on board BeppoSAX on 14 February 2000, 01:01:01 UT (Piro 2000). In the GRB monitor it exhibited a duration of $\sim 9 \mathrm{~s}$ and a $40-700 \mathrm{keV}$ fluence of $\sim 1.4 \times 10^{-5} \mathrm{erg} \mathrm{cm}^{-2}$. In the WFC $(2-30 \mathrm{keV})$, the duration was $\sim 115 \mathrm{~s}$ and the fluence $\sim 1.0 \times$ $10^{-6} \mathrm{erg} \mathrm{cm}^{-2}$ (Paolino et al. 2000). Follow-up observations with the BeppoSAX Narrow-Field Instrument (NFI) began about $12 \mathrm{~h}$ after the burst. A previously unknown X-ray fading point source, 1SAX J1854.4-6627, was detected in the MECS and LECS field of view at a position of R.A. $(\mathrm{J} 2000)=$ $18^{\mathrm{h}} 54^{\mathrm{m}} 27.0^{\mathrm{s}}$, Dec $(\mathrm{J} 2000)=-66^{\circ} 27^{\prime} 30^{\prime \prime}$ (error radius 50") with a $2-10 \mathrm{keV}$ flux of $5 \times 10^{-13} \mathrm{erg} \mathrm{cm}^{-2} \mathrm{~s}^{-1}$ (Antonelli et al. 2000a). Within the 50" radius of the NFI error circle, radio (Subrahmanyan et al. 2000) and IR (Rhoads et al. 2000) observations did not find any variable source. An estimation of the redshift based on the Fe $\mathrm{K} \alpha \mathrm{X}$-ray emission line yielded 0.37-0.47 (Antonelli et al. 2000a,b; Kotake \& Nagataki 2001).

Here we present optical observations of the GRB 000214 NFI error box in the UBVRI-bands, in order to search for objects with photometric redshifts in the range 0.37-0.47,

^ Based on observations obtained at the ESO $3.6 \mathrm{~m}$ telescope under ESO programme 165.H-0464(I). which could be potential candidates for the GRB 000214 host galaxy. Throughout, we assume a cosmology where $H_{0}=$ $65 \mathrm{~km} \mathrm{~s}^{-1} \mathrm{Mpc}^{-1}, \Omega_{\Lambda}=0$ and $\Omega_{\mathrm{M}}=1$.

\section{Observations}

All observations were obtained with the $3.6 \mathrm{~m}$ ESO telescope at La Silla (Chile). The CCD used was a Loral $2048 \times 2048$ detector giving a $5.4^{\prime} \times 5.4^{\prime}$ field of view. The observations were carried out in $2 \times 2$ binning mode, yielding a pixel scale of $0.31^{\prime \prime} /$ pixel. Table 1 displays the observing log. The photometry performed to study the content of the NFI error box is based on aperture photometry carried out using SExtractor (Bertin \& Arnouts 1996) to study the content of the NFI error box. The field was calibrated observing the Landolt star LTT 4816 (Landolt 1992) at an airmass similar to that of the GRB. Table 2 shows the positions and magnitudes of the selected secondary standards present in the NFI field (see Fig. 1).

\section{Results and discussion}

48 objects located closer than $1^{\prime}$ from the NFI position were detected in at least three optical bands (out of the five UBVRI filters). The magnitudes of these objects, and upper limits in the bands where no detection was possible, were used to feed 
Table 1. Journal of photometric observations of the GRB 000214 field with the $3.6 \mathrm{~m}$ ESO telescope. The magnitudes are given in the Vega system and are not corrected for Galactic reddening.

\begin{tabular}{lccccc}
\hline \hline Date UT & Filter & $\begin{array}{c}\text { Exp. time } \\
(\mathrm{s})\end{array}$ & $\begin{array}{c}\text { Seeing } \\
(\prime \prime)\end{array}$ & $\begin{array}{c}\text { Limiting mag. } \\
(3 \sigma)\end{array}$ & $\mathrm{AB}_{\text {off }}$ \\
\hline $23.3766 / 02 / 2002$ & $B$ & $3 \times 600$ & 1.3 & 24.1 & -0.071 \\
$23.3989 / 02 / 2002$ & $R$ & $2 \times 500$ & 1.1 & 23.4 & 0.222 \\
$24.3537 / 02 / 2002$ & $I$ & $3 \times 600$ & 1.1 & 22.2 & 0.449 \\
$24.3726 / 02 / 2002$ & $V$ & $2 \times 500$ & 1.1 & 24.3 & 0.045 \\
$24.3909 / 02 / 2002$ & $U$ & $2 \times 900$ & 1.0 & 22.8 & 0.732 \\
\hline
\end{tabular}

Table 2. Secondary standards in the field of GRB 000214.

\begin{tabular}{cccccccc}
\hline \hline & RA(J2000) & DEC (J2000) & $U$ & $B$ & $V$ & $R$ & $I$ \\
\hline A & $18: 54: 29.3$ & $-66: 28: 01$ & $17.25 \pm 0.01$ & $16.85 \pm 0.01$ & $16.62 \pm 0.01$ & $16.22 \pm 0.01$ & $15.71 \pm 0.01$ \\
B & $18: 54: 35.7$ & $-66: 28: 21$ & $18.62 \pm 0.01$ & $17.68 \pm 0.01$ & $17.17 \pm 0.01$ & $16.58 \pm 0.01$ & $15.96 \pm 0.01$ \\
C & $18: 54: 21.3$ & $-66: 27: 49$ & $19.15 \pm 0.01$ & $18.42 \pm 0.01$ & $18.01 \pm 0.01$ & $17.49 \pm 0.01$ & $16.98 \pm 0.01$ \\
\hline
\end{tabular}

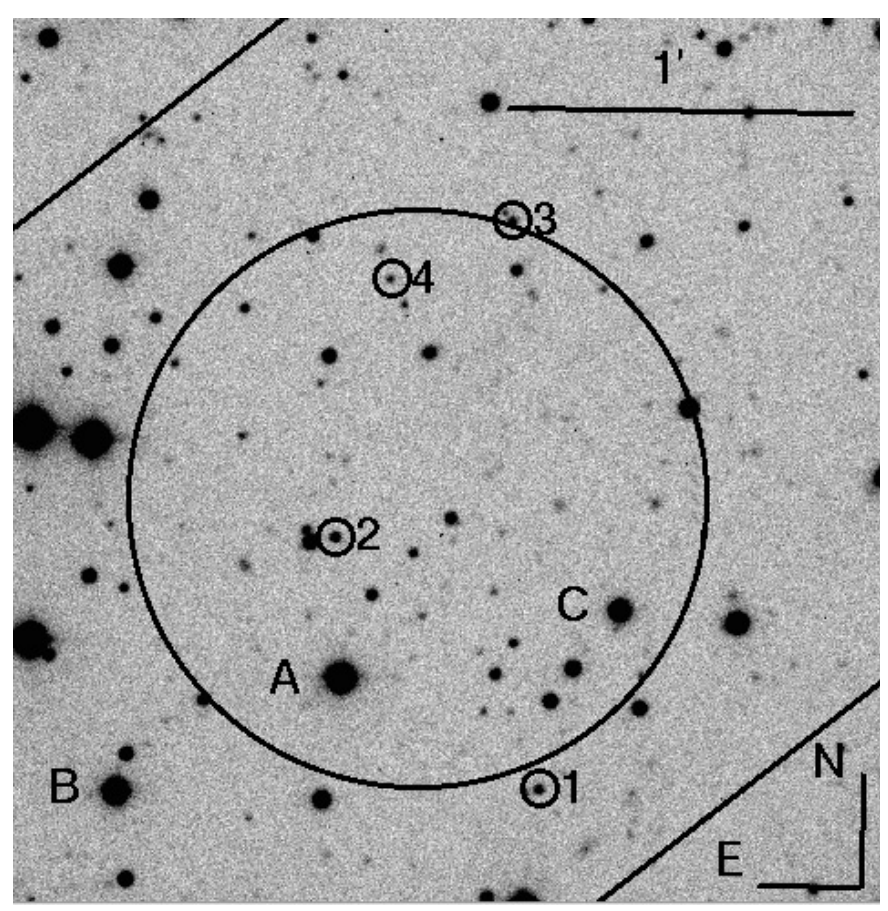

Fig. 1. $V$-band image obtained at the $3.6 \mathrm{~m}$ ESO telescope on 23 February 2002 of the GRB 000214 field. The BeppoSAX NFI error box (solid circle) of the GRB 000214 X-ray afterglow (Antonelli et al. 2000a) and a section of the IPN annulus (area inside the solid lines; Hurley \& Feroci 2000) are reported. The NFI error circle radius is $50^{\prime \prime}$ with a statistical confidence level of $90 \%$. The numbered objects show the potential candidates for the GRB 000214 host, displayed in Table 3. Candidate \#1 is slightly outside $\left(\sim 4.5^{\prime \prime}\right)$ and \#3 is on the edge of the NFI error circle. The stars labeled as A, B, C represent the secondary standards indicated in Table 2.

the HyperZ code, yielding the photometric redshift, extinction $\left(A_{\mathrm{v}}\right)$, galaxy type, and dominant stellar population age for each object (see Bolzonella et al. 2000 for more details on the HyperZ outputs). The photometric redshifts derived by HyperZ for GRB host galaxies have been tested in the past, using a sample of 10 hosts with known spectroscopic redshifts, and yielding excellent results, specially for GRB host galaxies classified as starbursts (see Table 2 of Christensen et al. 2004a). For the construction of the HyperZ synthetic templates, we assumed a Miller \& Scalo (1979) initial mass function, and a small Magellanic cloud (SMC) extinction law (Prevot et al. 1984), typical of GRB hosts galaxies.

Table 3 provides the coordinates, magnitudes, and photometric redshifts for our four best candidates. The photometric fluxes corresponding to our measurements were obtained convolving the $3.6 \mathrm{~m}$ ESO filter transmittances with the Loral $\mathrm{CCD}$, yielding the $\mathrm{AB}$ offsets $\left(\mathrm{AB}_{\text {off }}{ }^{1}\right)$ given in Table 1 .

Only object \#1 shows a photometric redshift fully consistent, within $1 \sigma$, with the $0.37-0.47$ redshift range, being the photometric redshift of candidate \#2 just at $1 \sigma$ from the X-ray redshift range lower limit. The two remaining objects (candidates \#3 and \#4) have photometric redshifts separated by $2 \sigma$ from the X-ray redshift range upper limit.

However, we note that candidate \#1 is formally outside of the $50^{\prime \prime}$ radius NFI error circle and object \#3 is just on its edge (see Fig. 1). Both candidates are fully consistent with the IPN annulus, so we decided not to discard them. Candidate \#2 is well centered in the NFI error circle, but its photometric redshift is only marginally consistent (at $1 \sigma$ ) with the X-ray redshift. Thus, inside the $90 \%$ confidence level NFI error box, no object has a photometric redshift fully consistent (within $1 \sigma$ ) with the $0.37-0.47 \mathrm{X}$-ray redshift range.

An alternative possibility is that the host galaxy of GRB 000214 is indeed placed within the $50^{\prime \prime}$ radius NFI error circle, but it is fainter in three or more filters than the limits reported in Table 1. In this case no computation of photometric redshift is possible and the object would be automatically discarded in our analysis. A second alternative scenario is possible if the GRB 000214 host galaxy is detected in three or more filters, but it is located on the outskirts of the NFI error circle (i.e. on the tail of the probability distribution). This might still be the case for object \#1, which is located only $\sim 4.5^{\prime \prime}$ out of the

1 The $\mathrm{AB}$ offset is defined as $\mathrm{AB}_{\text {off }}=-2.5 \log \left(F_{v}\right)-48.60-m_{\text {vega }}$, where $F_{v}$ is the flux density measured in erg s $\mathrm{sm}^{-1} \mathrm{~Hz}^{-1}$, and $m_{\text {vega }}$ is the magnitude in the Vega system. 
Table 3. Potential candidates for the host galaxy of GRB 000214. The table displays the coordinates, magnitudes, and inferred photometric redshifts for the four best host galaxy candidates. See Sect. 4 for a more extended description. The SEDs of these four objects can be seen in Fig. 3.

\begin{tabular}{ccccccccc}
\hline \hline$N$ & RA(J2000) & Dec (J2000) & $U$ & $B$ & $V$ & $R$ & $I$ & photo- $z$ \\
\hline 1 & $18: 54: 23.34$ & $-66: 28: 19.8$ & $>22.8$ & $23.34 \pm 0.11$ & $22.11 \pm 0.03$ & $21.09 \pm 0.04$ & $20.32 \pm 0.04$ & $0.49_{-0.07}^{+0.05}$ \\
2 & $18: 54: 29.34$ & $-66: 27: 36.7$ & $>22.8$ & $23.85 \pm 0.09$ & $21.77 \pm 0.02$ & $21.03 \pm 0.04$ & $20.49 \pm 0.04$ & $0.32_{-0.02}^{+0.05}$ \\
3 & $18: 54: 24.38$ & $-66: 26: 41.4$ & $>22.8$ & $23.06 \pm 0.11$ & $22.45 \pm 0.04$ & $21.58 \pm 0.11$ & $21.32 \pm 0.11$ & $0.53_{-0.03}^{+0.03}$ \\
4 & $18: 54: 27.87$ & $-66: 26: 51.8$ & $>22.8$ & $23.91 \pm 0.18$ & $23.11 \pm 0.06$ & $21.84 \pm 0.12$ & $>22.2$ & $0.57_{-0.04}^{+0.06}$ \\
\hline
\end{tabular}

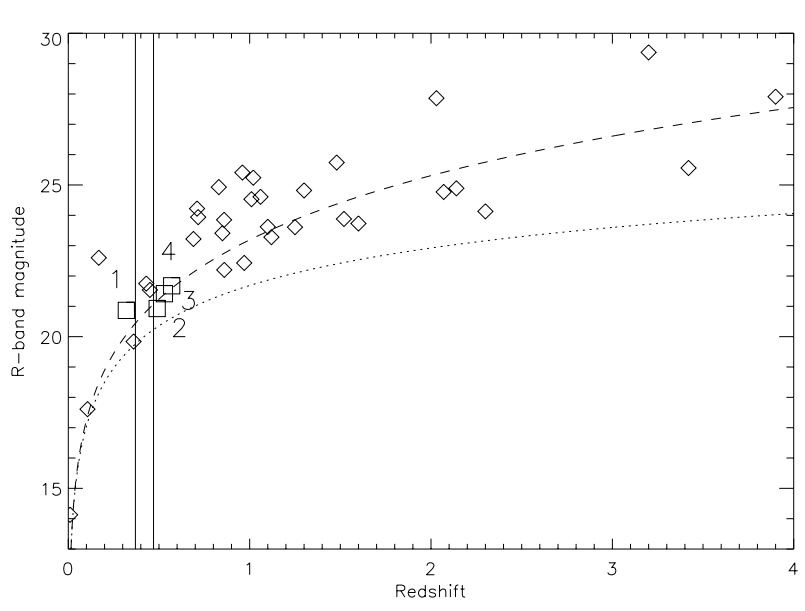

Fig. 2. The plot shows the $R$-band magnitude of 32 GRB host galaxies (rhomboids) and the potential four hosts of GRB 000214 (squares) as a function of redshift. All the magnitudes were corrected for Galactic reddening. The four candidates were labeled following the numeration given in Table 3. The error bars of our four candidates are smaller than the size of their symbols. The curves represent the $R$-band magnitude evolution of a typical $M_{R}^{\star}$ galaxy with redshift. The two vertical lines display the X-ray $0.37-0.47$ redshift bin. The K-corrections of the curves were carried out for two spectral indexes; $\beta=2$ (upper, dashed curve) and $\beta=0$ (lower, dotted curve). The area above both non-solid curves qualitatively indicates the region populated by subluminous galaxies. As can be seen, most of the hosts (including the four candidates) tend to be subluminous galaxies. The positions of the candidates are consistent with the loci of typical host galaxies at similar redshifts $(z \sim 0.5)$. All the magnitudes were corrected for foreground Galactic extinction, using the Schlegel et al. (1998) dust maps.

NFI error circle $90 \%$ boundary. The same conclusion stands for object \#3, which is just on the border of the NFI error circle.

In Fig. 2 we display the $R$-band magnitudes compiled for 32 host galaxies (rhomboids) and our four candidates (squares), once they are corrected for foreground Galactic extinction $(E(B-V)=0.061$, Schlegel et al. 1998). The curves display the apparent $R$-band magnitude of a reference $M_{R}^{\star}$ galaxy when it is redshifted from $z=0$ to $z=4$. $M_{R}^{\star}$ represents the $R$-band absolute magnitude and determines the knee of the luminosity function, separating the intrinsically bright from the subluminous galaxies. We assumed a value of $M_{R}^{\star}=-20.29+5 \log \left(H_{0} / 100\right)$ (Lin et al. 1996) estimated adopting an Einstein-de Sitter Universe (as in the present study). In order to perform the K-correction (Oke \& Sandage 1968), the spectrum of the $M_{R}^{\star}$ galaxy was assumed to be a power law $\left(F_{v} \sim v^{-\beta}\right)$, with the spectral index ranging from $\beta=0$ (lower dotted line) to $\beta=2$ (upper dashed line). This spectral index range generates a broad set of colours, $(0.3<B-R<1.2)$ accounting for most of the galaxies seen in the Hubble Deep Field (Williams et al. 1996). As shown, the four candidates seem to be subluminous galaxies, tending to be above the dotted curves. Thus, our candidates show apparent and absolute magnitudes similar to GRB host galaxies at similar redshifts $(z \sim 0.5)$.

The four objects were classified as starbursts by HyperZ, consistent with the hosts' photometric spectral energy distributions (SEDs) studied to date (Gorosabel et al. 2003a,b, 2005; Christensen et al. 2004a,b). The intrinsic extinction values of the host candidates range from $A_{\mathrm{v}}=0.0$ (objects \#3 and \#4) to $A_{\mathrm{v}}=2.85$ (object \#2), while object \#1 has an intermediate $A_{\mathrm{v}}$ value of 1.41 (see Fig. 3).

Three of our four candidates (\#1, \#2, and \#4) show compact appearance, at least under our seeing conditions (see Table 1), and display full width half maxima (FWHM) similar to other stellar objects present in the GRB field. Object \#3 is slightly extended in the images having the best seeing, so it very likely corresponds to a galaxy. The potential stellar nature of objects \#1, \#2, and \#4 was checked using the CLASS_STAR keyword given by SExtractor. Objects \#1, \#2, and \#4 show CLASS_STAR values below the mode of the CLASS_STAR distribution, specially in the $B$-band filter displaying CLASS_STAR $<0.8$. Systematically the object with the largest CLASS_STAR value is \#2. Therefore the four objects very likely correspond to galaxies, maybe with the exception of object \#2 whose stellar nature cannot be completely excluded.

One potential problem might be the presence of Active Galactic Nuclei (AGN) in our NFI error box, for which HyperZ (not accounting for emission due to a nebular component or/and a central massive compact source) would not be an appropriate tool to fit our SEDs. The expected number of AGNs brighter than $z=22.5$ (comparable to $R=23.4$, the $3 \sigma$ limit of our $R$-band image) and closer than $1^{\prime}$ from the NFI position is $\sim 1$ (Treister et al. 2004). Thus, for the sample of 48 objects, the AGN contamination is expected to be only $\sim 2 \%$.

Even for objects \#1 and \#2, which show the highest extinction among our four candidates $\left(A_{\mathrm{v}}=1.41\right.$ and $A_{\mathrm{v}}=$ $2.85 \mathrm{mag})$, their low photometric redshifts $(z=0.49$ and $z=0.32$ ) do not imply a high near-infrared restframe extinction. In particular the $K^{\prime}$-band limits imposed by Rhoads et al. (2000) would be only affected by intrinsic host extinctions of $A_{14400 \AA}=0.3$ and $A_{16300 \AA}=0.4$, for objects \#1 and \#2, respectively (assuming a SMC extinction law Prevot et al. 1984). 

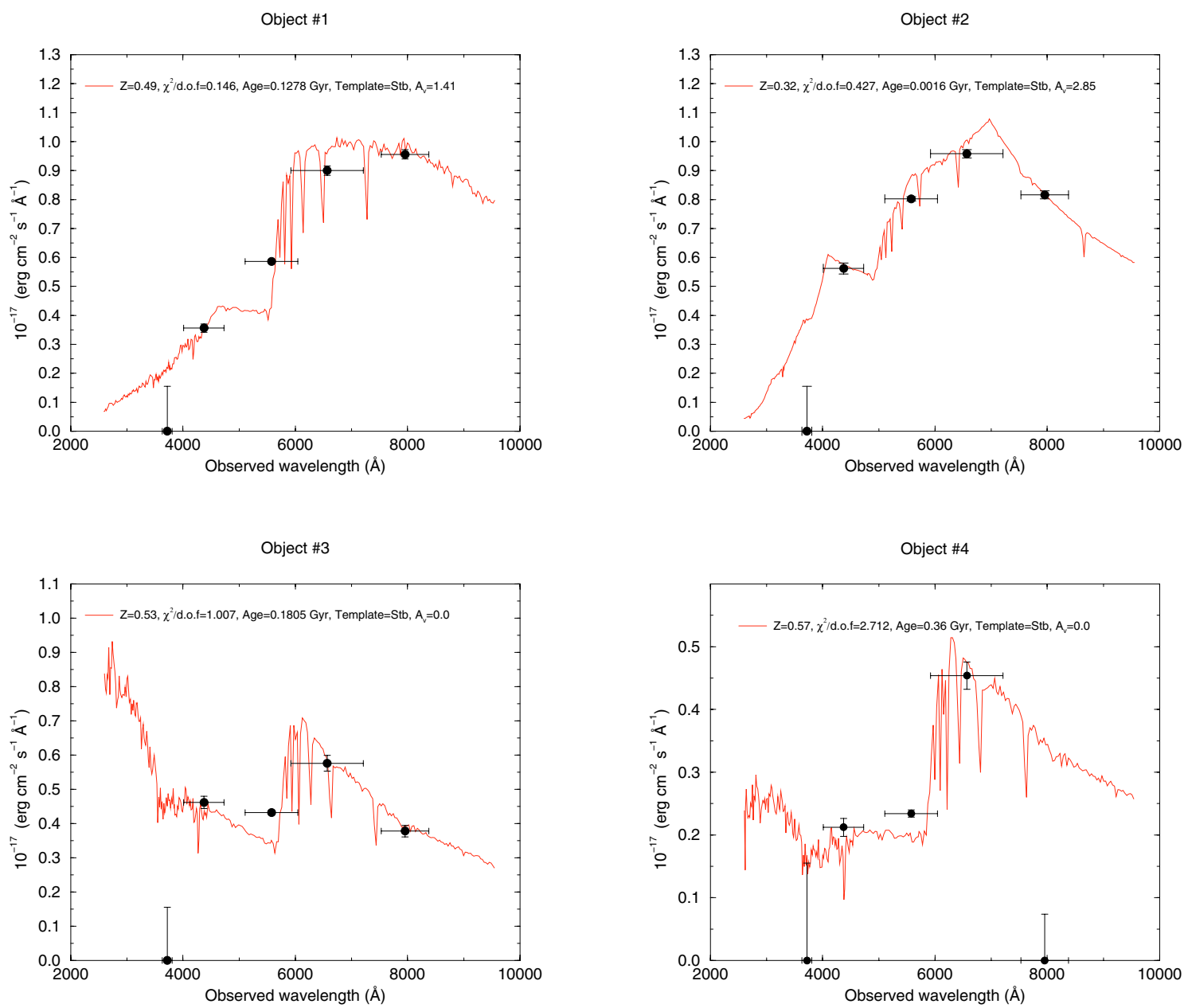

Fig. 3. Synthetic SEDs calculated by HyperZ for the four potential candidates for the GRB 000214 host galaxy. The synthetic spectra are shown by the line and the dots denote the UBVRI-band fluxes. The horizontal error bars indicate the FWHM of each filter. The detection upper limits are represented by vertical error bars ranging from the $3 \sigma$ limiting flux (see the associated limiting magnitude in the 5 th column of Table 1 ) to zero (a more detailed description of the upper limits used by HyperZ can be found in Bolzonella et al. 2000). The inferred values of the photometric redshift, age of the dominant stellar population, and extinction are given as inserts in the corresponding panels.

For objects \#3 and \#4, the $K^{\prime}$-band limit is even less extincted $\left(A_{\mathrm{v}} \sim 0\right)$. Therefore, if the host were one of our four objects, then it would be difficult to explain the $K^{\prime}$-band non-detection as an effect of the global intrinsic host extinction.

In fact, neither De Pasquale et al. (2003) nor Jakobsson et al. (2004) classify GRB 000214 as an intrinsically dark GRB. According to these authors the $K^{\prime}$-band and X-ray observations reported for this GRB are not fast/deep enough to constrain the physical parameters determining the SED.

\section{Conclusions}

We have here presented the result of $U B V R I$ photometry for all objects down to $R=23.4$ inside the GRB 000214 error box. After photometric reduction of the images and modeling of synthetic SEDs, we found no object within the $50^{\prime \prime}$ radius NFI error circle fully consistent with the redshift inferred from the X-ray spectrum.
However, we report four host galaxy candidates with photometric redshifts consistent within $2 \sigma$ with the $0.37-0.47 \mathrm{X}$-ray redshift range, so they are still statistically acceptable. Three of them are located inside (or just on the border of) the NFI error box, although they do not show photometric redshifts consistent (within $1 \sigma$ ) with the X-ray spectroscopic redshift range. A fourth $R=21.1$ mag object shows a photometric redshift of $z=0.49_{-0.07}^{+0.05}$, fully consistent within $1 \sigma$. We note that this candidate, although consistent with the IPN annulus, is slightly $\left(4.5^{\prime \prime}\right)$ outside of the $90 \%$ NFI error circle.

We cannot discard the idea that an object fainter (in three or more bands) than our UBVRI-band detection limits might be the actual GRB 000214 host galaxy. Further spectrophotometric observations of our four objects would definitively shed light on the reliability of the proposed candidates.

Acknowledgements. The data reported in the present paper were taken under the ESO programme 165.H-0464(I). We are grateful to the ESO staff at La Silla for performing the observations in the context of GRACE's host galaxy programme. S. Guziy acknowledges receipt of 
a fellowship grant from Spain's Ministerio de Ciencia y Tecnología (Ref. SB 2003-0236), and the hospitality at IAA-CSIC, where this research was carried out. J. Gorosabel acknowledges receipt of a Ramón y Cajal Fellowship from Spain's Ministerio de Ciencia y Tecnología. This research was partially funded by the Spanish ESP2002-04124C03-01 and AYA2004-01515 programmes (including FEDER Funds). We thank N. Masetti, E. Pian, and C. Kouveliotou for useful conversations. We acknowledge our anonymous referee for fruitful and constructive comments.

\section{References}

Antonelli, L. A., Piro, L., Tarei, G., et al. 2000a, GCN, 561 Antonelli, L. A., Piro, L., Vietri, M., et al. 2000b, ApJ, 545, L39 Bertin, E., \& Arnouts, S. 1996, A\&A, 117, 393

Bolzonella, M., Miralles, J.-M., \& Pelló, R. 2000, A\&A, 363, 476

De Pasquale, M., Piro, L., Perna, R., et al. 2003, ApJ, 592, 1018

Christensen, L., Hjorth, J., \& Gorosabel, J. 2004a, A\&A, 425, 913

Christensen, L., Hjorth, J., Gorosabel, J., et al. 2004b, A\&A, 413, 121
Gorosabel, J., Christensen, L., Hjorth, J., et al. 2003a, A\&A, 400, 127 Gorosabel, J., Klose, S., Christensen, L., et al. 2003b, A\&A, 409, 123

Gorosabel, J., Pérez-Ramírez, D., Sollerman, J., et al. 2005, A\&A, in press [astro-ph/0507488]

Hurley, K., \& Feroci, M. 2000, GCN, 556

Jakobsson, P., Hjorth, J., Fynbo, J. P. U., et al. 2004, ApJ, 617, L21

Kotake, K., \& Nagataki, S. 2001, PASJ, 53, 579

Landolt, A. U. 1992, AJ, 104, 372

Lin, H., Kirshner, R. P., Shectman, S. A., et al. 1996, ApJ, 470, 172

Miller, G. E., \& Scalo, J. M. 1979, ApJS, 41, 513

Oke, J. B., \& Sandage, A. 1968, ApJ, 154, 21

Paolino, A., Stornelli, Coletta, A., et al. 2000, GCN, 557

Piro, L. 2000, GCN, 550

Prevot, M. L., Lequeux, J., Prevot, L., et al. 1984, A\&A, 132, 389

Rhoads, J., Wilson, A., Storchi-Bergmann, T., et al. 2000, GCN, 564

Schlegel, D. J., Finkbeiner, D. P., \& Davis, M. 1998, ApJ, 500, 525

Subrahmanyan, R., Wieringa, M., Wark, R., et al. 2000, GCN, 562

Treister, E., Urry, C.M., Chatzichristou, E., et al. 2004, ApJ, 616, 123

Williams, R. E., Blacker, B., Dickinson, M., et al. 1996, AJ, 112, 1335 